\title{
Educação Física e o Fator Neurotrófico Derivado do Cérebro (BDNF) na Aprendizagem Escolar - Estudo de Revisão Sistemática
}

\author{
Jorge Marcos Ramos ${ }^{1}$ \\ Denival Soares Galdeano ${ }^{1}$
}

\section{RESUMO}

Objetivo: O objetivo dessa pesquisa foi fazer uma revisão sistemática para verificar a relação do exercício físico e o BDNF na aprendizagem. Como objetivos específicos: verificar qual o tipo de exercício é mais indicado; intensidade; relação com o gênero e com a faixa etária e ainda o melhor momento para sua realização. Metodologia: A busca pelos documentos foi realizada nas bases de dados: PubMed, LILACS e Google Acadêmico. Foram considerados artigos de revisão, experim entais e dissertações (Doutorado e Mestrado) publicados nos últimos vinte anos (1996 - 2016), escritos em inglês, espanhol e português. Como critérios de inclusão testes realizados em seres humanos e em modelos animais; relacionando à prática de exercícios com o BDNF e a sua influência na aprendizagem. Como critérios de exclusão os documentos que não apresentaram dados como: gênero, idade, intensidade, número e tempo de cada sessão. Foram selecionados 120 documentos, restando somente 19, segundo os critérios de inclusão. Resultado e Discussão: As pesquisas em neurociências relacionadas à educação contribuem para o aprimoramento das práticas pedagógicas levando em conta as funções encefálicas e suas relações com a aprendizagem. O Fator Neurotrófico Derivado do Cérebro (BDNF) tem como função promover a sobrevivência dos neurônios. O exercício físico promoveu alterações positivas nas concentrações de BDNF estando relacionado à melhora do desempenho neurológico. Conclusão: $O$ exercício aeróbio parece ser o mais indicado com intensidade vigorosa para o sexo masculino e a intensidade moderada para o feminino, as intervenções com tempo superiores há 20 minutos e com mais de uma sessão foram mais indicadas, contudo são necessárias novas pesquisas.

Palavras-chave: Educação física. Aprendizagem escolar. Atividade física.

\footnotetext{
${ }^{1}$ Faculdade de Ciências Médicas da Santa Casa de São Paulo. Departamento de Morfologia, São Paulo - SP, Brasil.
}

\section{Correspondência:}

Jorge Marcos Ramos. Secretaria Municipal de Educação de Santo André, Núcleo de Apoio a Natação Adaptada, Rua Marechal Hermes, 485, CEP 09090230, Jardim, Santo André - SP, Email: educacao.ramos@bol.com.br 


\section{Physical Education and the Brain Derived Neurotrophic Factor (BDNF) in School Learning - Systematic Review Study}

\section{ABSTRACT}

Objective: The aim of this research was to make a systematic review to verify the relationship between physical exercise and BDNF in learning. The specific aims were to verify which type of exercise is most appropriate; intensity; relationship with gender and age group, and also the best time to perform it. Methodology: Searches have been conducted in the follow databases: Pub Med, LILACS and Google Scholar. Experimental articles and dissertations ( $\mathrm{PhD}$ and Master's degrees) published in the last twenty years (1996 - 2016), written in English, Spanish and Portuguese were included. The following inclusion criteria were considered: tests performed in humans and animal models; relation of the practice of exercises with BDNF and its influence on learning. Documents that did not present data such as gender, age, intensity, number and time of each session have been excluded. 120 documents were identified, but only 19 fulfilled the inclusion criteria. Results and Discussion: Researches in neuroscience related to education contribute to improve the pedagogical practices taking into account brain functions and their relationships with learning. The Brain Derived Neurotrophic Factor (BDNF) has the function of promoting the neurons' survival. In general, the exercise promoted changes in BDNF concentration and was related to the improvement of neurological performance. Conclusion: Aerobic exercise was the most indicated with vigorous intensity for men and moderate intensity for women, intervention with more than 20 minutes and multiple sessions are more indicated.

Keywords: Physical Education. School learning. Physical Activity.

\section{Educación Física y el Factor Neurotrófico Derivado Del Cerebro (BDNF) en el Aprendizaje Escolar - Estudio de Revisión Sistemática}

\section{RESUMEN}

Objetivo: El objetivo de esta investigación fue hacer una revisión sistemática para verificar la relación del ejercicio físico y el BDNF en el aprendizaje. Como objetivos específicos: verificar qué tipo de ejercicio es más indicado; intensidad; relación conel género $y$ con el grupo de edad $y$ aún el mejor momento para su realización. Metodología: La búsqueda de los documentos se realizó en las bases de datos: PubMed, LILACS y Google Académico. Han sido considerados artículos de revisión, experimental y disertaciones (doctorado y máster) publicados en los últimos veinte años (1996-2016), escritos en Inglés, español y portugués. Como criterios de inclusión pruebas realizadas en seres humanos y en modelos animales; relacionando a la práctica de ejercicios con el BDNF y su influencia en el aprendizaje. Como criterios de exclusión los 
documentos que no presentaron datos como: género, edad, intensidad, número y tiempo de cada sesión. Fueran seleccionados 120 documentos, restando sólo 19 según los criterios de inclusión. Resultado y Discusión: Las investigaciones en neurociencias relacionadas a la educación contribuyen al perfeccionamiento de las prácticas pedagógicas teniendo en cuenta las funciones encefálicas y sus relaciones con el aprendizaje. El Factor Neurotrófico Derivado del Cerebro (BDNF) tiene como función promover la supervivencia de las neuronas. El ejercicio físico promovió cambios positivos en las concentraciones de BDNF estando relacionado a la mejora del desempeño neurológico.Conclusión: El ejercicio aeróbico fue el más indicado, con intensidad vigorosa para hombres y la intensidad moderada para mujeres, las intervenciones com tiempo superior a 20 minutos y con más de una sesión fu eron más indicadas, sin embargo se hace necesario nuevas investigaciones.

Palabras Clave: Educación Física. Aprendizaje escolar. Actividad Física. 


\section{INTRODUÇÃO}

Os avanços das pesquisas em neurociência relacionada à educação contribuem para o aprimoramento das práticas pedagógicas, pois levam em conta as funções encefálicas e suas relações com o processo de aprendizagem. A escola aparentemente baseia-se em um modelo de aprendizagem imóvel, sentada, mesmo sabendo que seu objetivo é promover o desenvolvimento global do educando, parece que o desenvolvimento intelectual é mais valorizado em detrimento aos demais como, por exemplo, a motricidade (GUERRA; LOPES; PEREIRA, 2004).

O currículo utilizado pela escola, a capacidade do professor, o método de ensino, o contexto da sala de aula, a família e a comunidade podem interferir no desenvolvimento neurológico do aluno e ainda, a falta de coordenação motora, do equilíbrio, da consciência espaço-temporal, dentre outras podem interferir na conduta desse aluno e consequentemente na sua aprendizagem (DA SILVA et al., 2007). O desenvolvimento motor abaixo do esperado não está associado exclusivamente às alterações neurológicas, pois esse desenvolvimento tem relação direta com a individualidade biológica como também é dependente das oportunidades oferecidas pelo ambiente (AMARO, 2010).

Em estudos utilizando modelo animal realizados por Knaepen et al. (2010), o exercício físico em particular os exercícios aeróbios demonstraram ser eficazes no aumento das concentrações do Fator Neurotrófico Derivado do Cérebro (Brain Derived Neurotrophic Fator - BDNF). O BDNF é uma proteína, membro da família das neurotrofinas, conforme Knaepen et al. (2010), e tem como funções promover a plasticidade sináptica, o crescimento axonal, a remodelação dendrítica e ainda de acordo com Rumajogee et al. (2004), a sobrevivência de vários neurônios do Sistema Nervoso Central (SNC).

Segundo Barde, Edgar e Thoenen (1982), o BDNF foi descoberto em 1982 por meio da purificação no cérebro de um porco, inicialmente o BDNF é sintetizado como pró-BDNF, sua forma precursora, que após sofrer ações enzimáticas dará origem ao BDNF maduro (mBDNF). Conforme Poo (2001), o mBDNF tem alta afinidade ao receptor tirosina cinase $B$ (TrkB) enquanto o pró-BDNF se liga ao receptor pan-neurotrofina 75(p75). O mBDNF é responsável pelo crescimento dos dendritos, sobrevivência celular e a formação do potencial de longa duração (Long Term Potentiation - LTP), enquanto que o pró-BDNF estimula à retração dendrítica, a depressão de longa duração (Long Term Depression- LTD) e a apoptose (POO, 2001).

O BDNF também tem funções importantes no Sistema Nervoso Periférico (SNP), pois estudos realizados por Pedersen et al. (2009) e Knaepen et al. (2010), em modelo animal, demonstraram que as concentrações reduzidas dessa proteína 
nessa região estão presentes em diversas doenças metabólicas e neurodegenerativas, tais como, a obesidade, o diabetes tipo II, a depressão, a doença de Alzheimer, a doença de Parkinson e o comprometimento cognitivo.

Segundo Zhao et al. (2004), o BDNF é encontrado com maior frequência no hipocampo, cerebelo e córtex cerebral, sendo produzido nos corpos celulares dos neurônios sensoriais, enquanto que no hipocampo é produzido predominantemente pelos dendritos pós-sinápticos. Sua síntese é regulada pelo aminoácido glutamato, cujo receptor N-Metil-D-Aspartano (NMDA) induz a LTP e causa uma rápida polimerização (formação de macromoléculas) de actina nas espinhas dendríticas dos neurônios do hipocampo conforme Aicard (2004), podendo ocorrer o bloqueio do aumento das espinhas dendríticas com a inibição da síntese do BDNF (TANAKA et al., 2008). Os neurônios não armazenam glicogênio, com isso para manter o fornecimento adequado de energia durante a ativação neuronal prolongada os astrócitos utilizam o lactado que é metabolizado na presença do BDNF (BROWN; TEKKOK; RANSOM, 2004).

Para Silvério e Rosat (2006), plasticidade neural é uma qualidade do Sistema Nervoso (SN) que consiste em desenvolver alterações nas funções sinápticas com o objetivo de prolongar ou facilitar a transmissão de um estímulo por meio da geração de traços de memória. As sinapses não são estáticas podendo modificar-se com o objetivo de tornar compatível sua atividade com a resposta esperada, isso é possível em decorrência das propriedades estruturais e moleculares que possibilitam aumentar a superfície de contato. Esse aumento na superfície de contato consiste em um mecanismo de base molecular no qual as alterações derivam da ativação de genes durante a passagem da memória denominada de curto prazo para a de longo prazo e da síntese de proteínas. A sensibilização dos receptores na membrana celular provoca o aumento intracelular do segundo mensageiro Adenosina Monofosfato Cíclico (AMPC) necessário para a ativação da cascata de proteína quinase (IZQUIERDO et al., 1998). Isso faz com que ocorra a transcrição do ácido ribonucleico (RNA) e síntese de novas proteínas específicas com o intuito de aumentar os contatos sinápticos (FLINT, 1999).

Conforme Rasmussen et al. (2009), em repouso ou durante a atividade física o SNC contribui significativamente com o BDNF circulante, essa proteína atravessa a unidade neurovascular e essa troca é feita em ambas as direções, com isso os níveis plasmáticos de BDNF podem ser utilizados como marcadores biológicos de alterações na memória e das funções cognitivas (KOMULAINEN et al., 2008; PAN, 1998). Também foi observado em estudo realizados por Hof e Morrison (2004) e Lista e Sorrentino (2010), relação negativa entre a idade (anos) e a expressão de BDNF e do seu receptor TrkB, pois quanto maior a idade (mais anos) menor é a produção de BDNF e consequentemente menor o número de conexões sinápticas.

Estudo realizado por Chaddock et al. (2010), sugerem que as alterações estruturais do encéfalo relacionadas ao exercício físico são melhores observados 
na região do hipocampo fazendo inferência de que o aumento no volume do hipocampo está diretamente relacionado à capacidade cognitiva e ao sucesso acadêmico (CHADDOCK et al., 2010; ERICKSON et al., 2011). De acordo com Morales-Mira e Maurício (2014), diversas moléculas podem mediar o efeito do exercício físico na neurogênese, sendo que a mais estudada é o BDNF e o Fator de Crescimento Endotelial Vascular (Vascular Endothelial Growth Factor - VEGF) estando diretamente relacionado ao aumento das conexões neuronais na região do hipocampo.

Segundo Best, White e Minai (2001), o hipocampo é um dos componentes do encéfalo humano localizado na base dos lobos temporais, direito e esquerdo apresentando complexas relações com outras regiões encefálicas, não sendo possível avaliar suas ações isoladamente em decorrência dessa vasta circuitaria. É considerado como a sede de produção da memória, estando envolvido no armazenamento temporário da informação e das memórias recentes e em fenômenos da formação do LTP. Em testes realizados em animais Burgess, Maguire e O'Keefe (2002) observaram que lesões no hipocampo resultam em profundas dificuldades na formação de novas memórias (amnésia anterógrada) e muitas vezes também afetam as memórias anteriores à lesão (amnésia retrógrada), sendo observado também que a inibição da proteína BDNF pode promover alterações negativas na formação do LTP (FIGUROV et al., 1996).

O hipocampo está interligado com diversas regiões do encéfalo, essas interações quando modificadas pela deterioração ou deficiência de neurotransmissores como ocorre no processo de senescência são associados à redução na capacidade de memorização (DELLA-MAGGIORE et al., 2000; LORINCZ; BUZSÁKI, 2000). A via serotoninérgica, por exemplo, quando estimulada pela serotonina aumentam o papel desses neurônios na memorização, conforme Williams, Rao e Goldman-Rakic (2002), já a acetilcolina está relacionada com o aumento da síntese do Fator de Crescimento Nervoso (Nerve Growth FatorNGV) e este com a consolidação da informação (WOOLF et al., 2001).

Estudos de neuro-imagem desenvolvidos por Bugg e Head (2011), em seres humanos sugerem que crianças e adultos com mais idade e com níveis de aptidão aeróbia mais elevada apresentam maior volume total do hipocampo em comparação com os seus pares sedentários. Um estilo de vida sedentário durante a infância não só influencia na saúde física, mas também na saúde encefálica, pois estudos realizados por Castelli et al. (2007) e Chaddock et al. (2010), demonstram que a redução na aptidão aeróbia está associada a baixos níveis de realização acadêmica, habilidades cognitivas, estrutura cerebral e funções encefálicas. 0 encéfalo humano apresenta de acordo com Rasmussen et al. (2009), produção de BDNF em repouso, contudo essa produção em repouso é aumentada quando o indivíduo realiza atividades aeróbias (SEIFERT et al., 2009).

A expressão do BDNF é regulada por outras variáveis fisiológicas, contudo 
como o objeto dessa pesquisa foi verificar o papel do exercício físico em relação ao BDNF iremos nos limitar a isso. A prática de exercício físico conforme Castelli et al. (2007), Rasmussen et al. (2009), Seifert et al. (2009), Chaddock et al. (2010) e Bugg e Head (2011), é um dos mecanismos que estimulam a síntese dessa proteína, seria adequado que os professores de educação física com base nessas informações utilizassem da sua prática para auxiliarem ou quem sabe potencializarem a aprendizagem de seus alunos em relação aos demais conteúdos curriculares e não somente aos referentes à sua disciplina, mesmo sabendo da pluralidade que podemos encontrar no ambiente escolar.

O objetivo dessa pesquisa foi fazer uma revisão sistemática para verificar a relação entre o exercício físico e o BDNF na aprendizagem escolar. Dentre os objetivos específicos podemos destacar: verificar qual o tipo de exercício físico é mais indicado (aeróbio ou anaeróbio); qual a intensidade a ser aplicada na realização do exercício físico (leve, moderada ou vigorosa); verificar se a relação entre exercício físico e o BDNF apresentam diferenças de acordo com o gênero (masculino ou feminino) do praticante como também no que diz respeito à faixa etária e por fim verificar qual o melhor momento para a realização do exercício físico (antes, durante ou após) em relação a aprendizagem de determinado conteúdo curricular da escola. Essa pesquisa se faz necessária tendo em vista a escassez de estudos que relacionam o efeito do exercício físico com o desenvolvimento neurológico em seres humanos. Algumas pesquisas descrevem alterações positivas na produção do BDNF em relação ao exercício físico, contudo ainda não está claro, em relação aos seres humanos, como o exercício físico deve ser utilizado para promover as alterações na produção dessa proteína.Com essas informações será possível subsidiar os professores de educação física para que possam qualificar suas ações para auxiliar os seus alunos no que se refere ao aprendizado dos demais componentes curriculares da escola, sem descaracterizar o papel fundamental dessa disciplina em relação ao desenvolvimento motor.

\section{MÉTOdo}

Esta pesquisa se caracterizou como uma revisão sistemática, visando discorrer sobre o papel do exercício físico e o Fator Neurotrófico Derivado do Cérebro (BDNF) durante o processo de aprendizagem dos demais conteúdos curriculares desenvolvidos no ambiente escolar.

A busca pelos documentos foi realizada nas bases de dados: PubMed, LILACS e Google Acadêmico, sendo utilizada a combinação das palavras-chaves: educação física, fator neurotrófico derivado do cérebro (BDNF), aprendizagem, sala de aula, atividade física, exercício físico, cognição e memória.

Foram considerados artigos de revisão, artigos experimentais, dissertações 
(Doutorado e Mestrado) publicados nos últimos vinte anos (1996-2016), escritos em inglês, espanhol e português. Esse intervalo para a seleção dos documentos se fez necessário, tendo em vista a escassez de pesquisas que relacionam a prática de exercícios físicos com o desenvolvimento encefálico na aprendizagem escolar.

Como critérios de inclusão os artigos deveriam referir-se a testes realizados com seres humanos como também em modelos animais, tendo em vista o maior número de pesquisas. Os documentos deveriam relacionar à prática de exercícios físicos com o BDNF e a sua influência frente à aprendizagem escolar. Como critérios de exclusão os documentos que não apresentaram dados relevantes para a pesquisa como: gênero dos participantes, idade, intensidade do exercício físico, número de sessões e o tempo destinado para cada sessão foram descartados.

Inicialmente foram selecionados 120 documentos, após análise somente 19 obedeceram aos critérios de inclusão sendo os demais descartados, conforme a Figura 1:

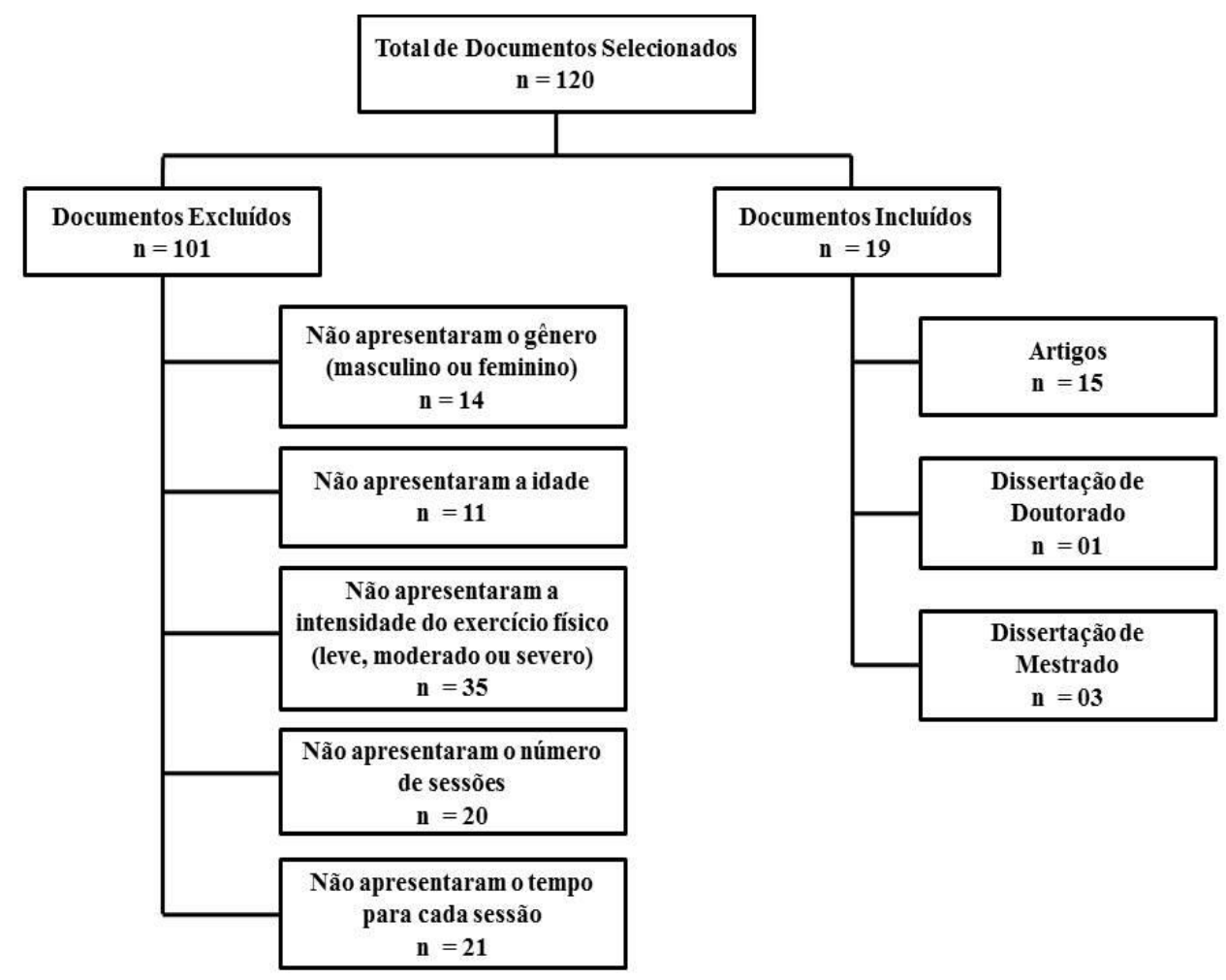

Figura 1 - Total de documentos selecionados: documentos excluídos e documentos incluídos.

\section{REsultados E Discussão}

Dentre os documentos selecionados, somente 19 estavam de acordo com os critérios de inclusão. Os documentos que obedeceram estes critérios foram lidos 
novamente e deles extraídos os dados relevantes para essa pesquisa conforme disposto no Quadro 1:

\section{Quadro 1 - Caracterização da amostra: Dados dos documentos selecionados para a pesquisa: gênero, idade, intensidade do exercício, número de sessões e resultados encontrados}

\begin{tabular}{|c|c|c|c|c|c|c|c|}
\hline No & Autor (S) & $\begin{array}{l}\text { Ano } \\
\text { Publ. }\end{array}$ & $\begin{array}{c}\text { Amostra } \\
(\mathrm{M} / \mathrm{F})\end{array}$ & $\begin{array}{l}\text { Idade dos } \\
\text { Participantes } \\
\quad \text { (anos) }\end{array}$ & $\begin{array}{l}\text { Intensidade } \\
\text { Exercício }\end{array}$ & $\begin{array}{c}\text { No sessões } \\
\text { X } \\
\text { Tempo de } \\
\text { cada } \\
\text { sessão } \\
\text { (minutos) }\end{array}$ & Resultado \\
\hline 01 & $\begin{array}{l}\text { Ardoy } \\
\text { et al. }\end{array}$ & 2014 & $\begin{array}{l}M-43 \\
F-24 \\
67\end{array}$ & $12-14$ & $\begin{array}{l}\text { Moderada } \\
\quad \text { a } \\
\text { Vigorosa }\end{array}$ & $\begin{array}{c}2 \text { ou } 4 \\
\text { sessões } \\
55 \text { minutos }\end{array}$ & $\begin{array}{l}\text { Duplicar o número de aulas de } \\
\text { Educação Física não apresentou } \\
\text { alterações no desempenho cognitivo, } \\
\text { porém ao aumentar a intensidade dos } \\
\text { exercícios foi observado melhora em } \\
\text { relação ao desempenho cognitivo. }\end{array}$ \\
\hline 02 & Bakshi & 2008 & $\begin{array}{c}\text { M- } 13 \\
F-7 \\
20 \\
\end{array}$ & $20-22$ & $\begin{array}{l}\text { Moderada } \\
\text { a } \\
\text { Vigorosa }\end{array}$ & $\begin{array}{l}5 \text { sessões } \\
30 \text { minutos }\end{array}$ & $\begin{array}{l}\text { A atividade física melhorou a } \\
\text { aprendizagem da matemática e inglês. }\end{array}$ \\
\hline 03 & $\begin{array}{l}\text { Beck } \\
\text { et al. }\end{array}$ & 2016 & $\begin{array}{l}\text { M- } 88 \\
\text { F- } 77 \\
165\end{array}$ & $6-8$ & $\begin{array}{l}\text { Leve } \\
\quad \text { a } \\
\text { Moderada }\end{array}$ & $\begin{array}{l}18 \text { sessões } \\
60 \text { minutos }\end{array}$ & $\begin{array}{c}\text { Enriquecimento motor melhorou o } \\
\text { desempenho na aprendizagem da } \\
\text { matemática (atividade motora grossa } \\
\text { foi mais significativa) }\end{array}$ \\
\hline 04 & Chaddock & 2013 & $\begin{array}{c}\text { M- } 11 \\
\text { F- } 17 \\
28 \\
\end{array}$ & $8-9$ & $\begin{array}{l}\text { Moderada } \\
\text { a } \\
\text { Vigorosa }\end{array}$ & $\begin{array}{c}180 \\
\text { sessões } \\
15 \text { minutos } \\
\end{array}$ & $\begin{array}{c}\text { Sugere melhora em relação à atenção e } \\
\text { aumento do volume dos gânglios da } \\
\text { base e do hipocampo. }\end{array}$ \\
\hline 05 & $\begin{array}{l}\text { Chan } \\
\text { et al. }\end{array}$ & 2008 & $\begin{array}{c}M-30 \\
F-30 \\
60 \\
\end{array}$ & $6-10$ & Moderada & $\begin{array}{l}40 \text { sessões } \\
30 \text { minutos }\end{array}$ & $\begin{array}{l}\text { Melhora na memória e na } \\
\text { aprendizagem. }\end{array}$ \\
\hline 06 & $\begin{array}{l}\text { De } \\
\text { Moraes } \\
\text { Ferrari } \\
\text { et al. }\end{array}$ & 2014 & $\begin{array}{l}\text { M- } 119 \\
\text { F- } 107 \\
226\end{array}$ & $8-18$ & Vigorosa & $\begin{array}{l}1 \text { sessão } \\
30 \text { minutos }\end{array}$ & $\begin{array}{c}\text { Melhor condição físicarefletiu em } \\
\text { melhora na aprendizagem da disciplina } \\
\text { de ciências }\end{array}$ \\
\hline 07 & $\begin{array}{l}\text { Dias } \\
\text { et al. }\end{array}$ & 2013 & $\begin{array}{c}\text { M- } 22 \\
\text { F- } 21 \\
43 \\
\end{array}$ & $9-10$ & Moderada & $\begin{array}{l}1 \text { sessão } \\
30 \text { minutos }\end{array}$ & Melhorou o desempenho acadêmico \\
\hline 08 & Epstein & 2011 & $\begin{array}{c}M-40 \\
F-92 \\
132\end{array}$ & $20-24$ & $\begin{array}{l}\text { Moderada } \\
\text { a } \\
\text { Vigorosa } \\
\end{array}$ & $\begin{array}{l}1 \text { sessão } \\
25 \text { minutos }\end{array}$ & Melhorou a atenção \\
\hline 09 & $\begin{array}{l}\text { Erickson } \\
\text { et al }\end{array}$ & 2011 & $\begin{array}{l}M-0 \\
F-120 \\
120 \\
\end{array}$ & $60-69$ & Moderada & $\begin{array}{l}20 \text { sessões } \\
40 \text { minutos }\end{array}$ & Aumentou o volume do Hipocampo \\
\hline 10 & Halvorson & 2014 & $\begin{array}{l}\text { M- } 15 \\
\text { F- } 6 \\
21 \\
\end{array}$ & $6-11$ & Moderada & $\begin{array}{l}1 \text { sessão } \\
20 \text { minutos }\end{array}$ & $\begin{array}{l}\text { Não houve diferença significativa, } \\
\text { contudo, o tempo de reação foi mais } \\
\text { lento para o grupo sedentário. }\end{array}$ \\
\hline 11 & $\begin{array}{l}\text { Hopkins } \\
\text { et al. }\end{array}$ & 2012 & $\begin{array}{c}\text { M- } 40 \\
\text { F- } 14 \\
54\end{array}$ & $18-36$ & Moderada & $\begin{array}{l}20 \text { sessões } \\
30 \text { minutos }\end{array}$ & $\begin{array}{l}\text { Foi observado melhora na cognição e } \\
\text { bem-estar psicológico. }\end{array}$ \\
\hline 12 & $\begin{array}{l}\text { Labban } \\
\text { e } \\
\text { Etnier }\end{array}$ & 2011 & $\begin{array}{c}M-20 \\
F-44 \\
64\end{array}$ & $18-34$ & Moderada & $\begin{array}{l}2 \text { sessões } \\
30 \text { minutos }\end{array}$ & $\begin{array}{c}\text { O grupo que realizou exercício antes da } \\
\text { atividade cognitiva apresentou melhora } \\
\text { na memória de trabalho. }\end{array}$ \\
\hline 13 & $\begin{array}{l}\text { Reed } \\
\text { et al. }\end{array}$ & 2013 & $\begin{array}{c}\text { M- } 236 \\
F-234 \\
470\end{array}$ & $7-13$ & Moderado & $\begin{array}{c}1 \text { ou } 5 \\
\text { sessões } \\
45 \text { minutos }\end{array}$ & Melhorou a capacidade cognitiva \\
\hline 14 & $\begin{array}{c}\text { Schmidt- } \\
\text { Kassow } \\
\text { et al. }\end{array}$ & 2010 & $\begin{array}{c}M-4 \\
F-8 \\
12 \\
\end{array}$ & $19-33$ & Moderada & $\begin{array}{l}3 \text { sessões } \\
30 \text { minutos }\end{array}$ & $\begin{array}{l}\text { Atividade simultânea (ciclismo) facilitou } \\
\text { a memorização de novos itens. }\end{array}$ \\
\hline 15 & Skrade & 2013 & $\begin{array}{c}\text { M- } 152 \\
F-132 \\
284\end{array}$ & $9-11$ & Moderada & $\begin{array}{l}8 \text { sessões } \\
10 \text { minutos }\end{array}$ & $\begin{array}{l}\text { Melhora na aprendizagem da } \\
\text { matemática. }\end{array}$ \\
\hline 16 & $\begin{array}{l}\text { Skriver } \\
\text { et al. }\end{array}$ & 2014 & $\begin{array}{c}M-32 \\
F-0 \\
32\end{array}$ & $18-35$ & Vigorosa & $\begin{array}{l}1 \text { sessão } \\
20 \text { minutos }\end{array}$ & $\begin{array}{l}\text { Foi encontrado alterações positivas em } \\
\text { relação à memória e aprendizagem. }\end{array}$ \\
\hline 17 & $\begin{array}{l}\text { Spitzer e } \\
\text { Hollmann }\end{array}$ & 2013 & $\begin{array}{c}M-28 \\
F-16 \\
444\end{array}$ & $12-13$ & $\begin{array}{c}\text { Moderada } \\
\text { a } \\
\text { Vigorosa }\end{array}$ & $\begin{array}{l}30 \text { sessões } \\
30 \text { minutos }\end{array}$ & $\begin{array}{l}\text { Melhorou a aprendizagem da } \\
\text { matemática. }\end{array}$ \\
\hline 18 & $\begin{array}{c}\text { Van Den } \\
\text { Berg } \\
\text { et al. }\end{array}$ & 2016 & $\begin{array}{c}\text { M- } 103 \\
\text { F- } 81 \\
184\end{array}$ & $10-13$ & $\begin{array}{c}\text { Leve } \\
\text { a } \\
\text { Moderada }\end{array}$ & $\begin{array}{l}1 \text { sessão } \\
12 \text { minutos }\end{array}$ & $\begin{array}{c}\text { Não houve alteração em relação ao } \\
\text { desempenho cognitivo. }\end{array}$ \\
\hline 19 & $\begin{array}{l}\text { Winter } \\
\text { et al. }\end{array}$ & 2007 & $\begin{array}{c}\text { M- } 27 \\
F-0 \\
27 \\
\end{array}$ & $19-27$ & $\begin{array}{c}\text { Moderada } \\
\text { a } \\
\text { Vigorosa }\end{array}$ & $\begin{array}{l}1 \text { sessão } \\
40 \text { minutos }\end{array}$ & $\begin{array}{l}\text { A atividade vigorosa apresentou } \\
\text { melhora no desempenho cognitivo. }\end{array}$ \\
\hline
\end{tabular}


As alterações estruturais do encéfalo relacionados em especial a prática de exercícios físicos são melhores observados na região do hipocampo, em relação a essas alterações podemos destacar os estudos de Erickson et al. (2011) e Chaddock (2013), os quais observaram aumento do volume do hipocampo e dos núcleos da base em decorrência da pratica de exercícios físicos. Para os autores esse aumento proporcionou melhora na capacidade de memorização e na atenção, respectivamente. Para Erickson et al. (2011), o aumento no volume do hipocampo está associado a maiores níveis séricos de BDNF relacionados à pratica de exercícios físicos, contudo devemos destacar que nesses dois estudos as faixas etárias eram distintas (crianças 8-9 anos x idosos 60-69 anos) e em ambos foram observados aumentos em relação ao volume do hipocampo, o que é benéfico para todas as faixas etárias tendo em vista o papel fundamental dessa estrutura encefálica em relação à formação da memória.

Com o objetivo de avaliar alterações na capacidade de memorização (memória) Chan et al. (2008) e Skriver et al. (2014), relataram que a prática de exercício físico proporcionou melhora na aprendizagem em decorrência da melhora na formação da memória dos participantes. Skriver (2014), descreve que após a prática de exercícios físicos foram avaliados os níveis sanguíneos de biomarcadores em particular o BDNF e o lactado, sendo que o grupo de exercício físico apresentou maiores níveis dessas substâncias como também um melhor desempenho no teste de memória.

Procurando mostrar a importância das aulas de educação física no contexto escolar De Moraes Ferrari et al. (2014), avaliaram se a prática dessa disciplina influenciava no desempenho do aluno nas demais disciplinas, sendo encontrado efeitos positivos em relação ao condicionamento físico e o desempenho na disciplina de Ciências. Nessa mesma linha Bakshi (2008), comparou a prática da educação física tradicional com a prática do crossfit kids, na aprendizagem da matemática sendo observado melhora no desempenho da matemática para os praticantes do crossfit kids. Spitzer e Hollmann (2013), também observaram melhora na aprendizagem da matemática para os participantes de exercícios físicos.

Em outro modelo de exercício físico denominado "movimento para o pensamento" que consistia em 10 movimentos realizados na sala de aula Skrade (2013), comparou com o processo tradicional de desenvolvimento das aulas de educação física, sendo encontradas alterações positivas em relação à aprendizagem da matemática para àqueles que participaram do novo modelo de exercício físico. Avaliando ainda o desempenho na matemática Beck et al. (2016), compararam o desempenho na aprendizagem da matemática em três grupos: matemática e atividade motora fina; matemática e atividade motora grossa e matemática no processo de ensino tradicional. O estudo demonstrou que o aprendizado da matemática com enriquecimento motor foi aumentado e ainda que 
as atividades motoras grossas foram mais significativas no processo de aprendizagem da referida disciplina. Corroborando com esses achados Dias et al. (2013), avaliaram o efeito de brincadeiras ativas (pega-pega) nas aulas de educação física, constatando melhora no desempenho relacionado à atenção seletiva.

Em relação ao aprimoramento da cognição Winter et al. (2007), avaliaram somente indivíduos do sexo masculino, sendo observado aumento de $20 \%$ na aprendizagem de vocábulos para o grupo que realizou exercícios físicos vigorosos. Nessa mesma linha Hopkins et al. (2012), identificaram que a prática de exercícios físicos moderado melhorou o desempenho cognitivo dos participantes. Ainda em relação a cognição Reed et al. (2013), avaliaram o efeito da educação física realizada uma vez por semana e/ou cinco vezes por semana, sendo observado que a prática de educação física mais vezes na semana com a mesma intensidade pode melhorar a capacidade cognitiva, sendo essas alterações mais evidenciadas no grupo feminino.

Em relação ao número de sessões semanais e sua relação com a intensidade (leve, modera ou vigorosa) Ardoy et al. (2014), avaliaram três grupos, sendo que o grupo 01 realizou duas aulas semanais de educação física, o grupo02 realizou quatro aulas semanais e o grupo 03 também realizou quatro aulas semanais em alta intensidade. Foram observados que duplicar o número de aulas de educação física não apresentou melhoras significativas no desempenho cognitivo, apresentando melhoras em outras variáveis fisiológicas, no entanto em relação ao aumento da intensidade foi observado melhora significativa em relação ao desempenho cognitivo e acadêmico em especial dentre os participantes do sexo masculino. Epstein (2011) e Halvorson (2014), em seus ensaios não encontraram alterações em relação à cognição, no entanto descrevem que foram observadas alteração positivas em relação à atenção e ao tempo de reação, respectivamente.

Avaliando o incremento de movimentos corporais durantes as aulas convencionais que compõe o currículo escolar, fazendo com que durante as aulas na sala os alunos não permanecessem todo o tempo sentado. Para tanto, os participantes realizaram exercício físico denominado "exercício mente e corpo", composto por 13 movimentos que tinham por objetivo melhorar o desempenho acadêmico, problemas comportamentais, emocionais e a memória. Foi observado que as crianças do grupo experimental apresentaram alterações positivas em relação ao comportamento, à memória e ao desempenho acadêmico (CHAN; CHEUNG; SZE, 2008).

Em relação ao momento ideal para a prática do exercício físico: antes, durante ou após o conteúdo a ser aprendido Schmidt-Kassow et al. (2010), observaram melhora significativa em relação à aprendizagem (memorização) para o grupo que realizou exercício físico durante a leitura de palavras. Nessa mesma linha Labban e Etnier (2011), constituíram três grupos: 1- Exercício antes do texto 
a ser memorizado; 2- Exercício depois da leitura do texto a ser memorizado e 3Memorização do texto sem a prática de exercício físico, segundo os autores somente o grupo que realizou exercício físico antes da leitura do texto apresentou melhora significativa na memória de trabalho, sugerindo que o momento para a realização do exercício pode modular o seu efeito frente à aprendizagem, no entanto se faz necessário novos estudos, pois as pesquisas são contraditórias.

E por fim se faz necessário avaliar também qual o tipo de exercício físico apresenta melhor eficiência em relação ao desenvolvimento neurológico, para tanto foi realizado um estudo que tinha por objetivo testar três tipos de exercícios: aeróbios, de coordenação e de força, realizados na sala de aula. Foi aplicado um teste pré e pós-intervenção relacionado à cognição, não sendo encontradas diferenças significativas em nenhum dos tipos de exercícios (VAN DEN BERG et al., 2016).

O objetivo dessa pesquisa foi verificar a relação da prática de exercícios físicos com o BDNF na aprendizagem escolar, procurando identificar qual o tipo de exercício físico é mais indicado (aeróbio ou anaeróbio); em qual intensidade esse exercício físico deve ser realizado; se existe diferença para a realização do exercício físico em relação ao gênero dos participantes como também em relação à faixa etária e ainda em qual momento da aprendizagem o efeito do exercício físico se torna mais adequado.

O que é possível observar no dia a dia da escola é que na sala de aula o encéfalo é estimulado recebendo diversas informações e o corpo deve ficar imóvel, ocorrendo uma separação equivocada em que o desenvolvimento relativo ao gesto motor está a cargo da educação física e o desenvolvimento cognitivo sendo de responsabilidade das demais disciplinas que compõe o currículo escolar. Estudos realizados por Castelli et al. (2007) e Chomitz et al. (2009), observaram que uma menor aptidão aeróbia está associada a uma diminuição na aprendizagem da leitura e da matemática, sendo sugerido que dispor de mais tempo para a prática de exercício físico pode melhorar o desempenho na aprendizagem dessas disciplinas (HILLMAN; ERICKSON; KRAMER, 2008).

Segundo Knaepen et al. (2010) a comparação entre os estudos relacionando o exercício físico e o aumento do BDNF é muito difícil em decorrência das diferentes populações estudadas, o tamanho das amostras, a utilização de diferentes protocolos em relação ao exercício físico, a utilização de diferentes intensidades, dentre outras variáveis, e essa dificuldade mostrou-se nessa pesquisa, pois dentre os documentos selecionados as faixas etárias são diferentes e mesmo quando foram observados ensaios em que as faixas etárias são mais próximas a intensidade aplicada aos exercícios físicos são distintas.

O que se pode apresentar é que a prática de exercício físico promove alterações na produção do BDNF e que o exercício aeróbio parece ser mais indicado 
para esse aumento, tendo em vista que dentre os 19 documentos avaliados somente o de número 18, Van Den Berg et al. (2016) não encontrou alteração cognitivas após a prática de exercícios físicos, nessa pesquisa em especial vale ressaltar que foi a única dentre as selecionadas que utilizou o exercício físico em uma intensidade leve a moderada, todos os outros utilizaram uma intensidade de moderada para vigorosa, podendo ser essa variável a responsável por esse resultado diferente.

Nas aulas de educação física seria adequado que os professores responsáveis por essa disciplina tivessem maiores informações em relação ao que está sendo desenvolvido na sala de aula para que pudessem desenvolver em suas aulas atividades com essa perspectiva (SZUHANY; BUGATTI; OTTO, 2015). Em relação ao número de sessões (aulas semanais) Ardoy et al. (2014), observaram que o simples fato de aumentar de 2 para 4 aulas semanais não alterou a qualidade cognitiva, contudo quando nessas 4 aulas foram aumentadas a intensidade dos exercícios físicos ocorreram melhoras significativas na cognição dos avaliados.

Em relação ao número de sessões segundo Van Den Berg et al. (2016), parece haver uma relação dependente entre essas variáveis, pois as sessões com tempo superiores a 20 minutos de acordo com Reed et al. (2013), e com mais de uma sessão promoveram alterações positivas nos testes de memória. Com esse propósito Mota et al. (2015), recomenda 40 minutos diários de atividade física por 3 dias na semana e ainda com intensidade vigorosa. Em outros estudos Skriver et al. (2014), So (2012) e Winter et al. (2007), observaram que a intensidade vigorosa é mais indicada para os praticantes do sexo masculino e a intensidade moderada, segundo So (2012), promovem melhores alterações para os praticantes do sexo feminino. Corroborando com esses achados foi realizada uma meta-análise que avaliou 29 estudos relacionados aos efeitos do exercício físico sobre o BDNF, sendo identificado que estudos com mais mulheres apresentaram menores alterações no BDNF em relação a pratica de exercícios físicos, tendo em vista que a intensidade utilizada nessas pesquisas foi a vigorosa conforme Szuhany, Bugatti e Otto (2015), sendo essa intensidade recomendada para os praticantes do sexo masculino (SKRIVER et al., 2014; SO, 2012; WINTER et al., 2007). Vale ressaltar que essa meta-analise apresentou as mesmas dificuldades em relação as faixas etárias como também em relação às outras variáveis.

Dentre as faixas etárias estudadas, os adultos jovens estão no auge da sua capacidade cognitiva e isso talvez interfira na influência do exercício físico nessa região, não sendo possível atribuir somente a este as melhoras relacionadas às habilidades cognitivas quando comparados a outros adultos jovens sedentários de acordo com Skriver (2014), sendo necessários novos estudos com um maior rigor em relação ao controle de determinadas variáveis como quantidade e qualidade do sono e alimentação, pois estas também interferem na produção do BDNF.

E por fim Schmidt-Kassow et al. (2010) realizaram um teste utilizando à 
prática de exercício físico simultâneo à aprendizagem de palavras sendo observado melhora significativa para o grupo que praticou exercício físico e ouviu uma lista de palavras, simultaneamente. Contudo outro estudo realizado por Labban e Etnier (2011) mostrou que o momento ideal para a prática de exercício físico seria antes da leitura do texto, com isso a memorização se tornaria mais efetiva evidenciando com isso o papel modulador do exercício físico em relação à produção e utilização do BDNF.

Outro modelo de intervenção avaliado por Chan, Cheung e Sze (2008) foi o enriquecimento motor realizado durante as aulas tradicionais, mais uma vez a intensidade dos exercícios como também o número de sessões parece interferir no resultado final, pois nos estudos que utilizaram mais tempo (minutos) e maior número de sessões os resultados apresentaram relação positiva com 0 desenvolvimento cognitivo.

Essa pesquisa não tem a pretensão de encerrar as discussões em relação a esse tema tão importante para a educação física, sendo necessários outros estudos para fortalecer os achados em relação aos benefícios dos exercícios físicos e o desenvolvimento neurológico, contudo diante do que se sabe a esse respeito seria adequado que os professores de educação física na escola tivessem maiores informações em relação aos demais conteúdos que estão sendo apresentados a esses alunos para que pudessem colaborativamente elaborar as suas aulas com esse olhar em relação ao BDNF e se possível romper essa relação equivocada onde o desenvolvimento cognitivo fica a cargo dos conteúdos desenvolvidos na sala de aula e o desenvolvimento motor a cargo da educação física.

\section{CONSIDERAÇÕES FINAIS}

Foi possível observar dentre os artigos analisados que o exercício físico promove alterações positivas nas concentrações de BDNF estando relacionado a melhorado desempenho neurológico dos participantes.

O exercício aeróbio parece ser mais indicado, sendo que os exercícios físicos com intensidade moderada à vigorosa apresentaram melhores resultados em relação ao desempenho cognitivo, no entanto para o sexo feminino a intensidade moderada se mostrou mais indicada e para o sexo masculino foi à vigorosa.

De maneira geral foi indicado intervenções com tempo superiores há 20 minutos e com mais de uma sessão, contudo se faz necessário novas pesquisas com um melhor controle em relação à faixa etária e ao momento ideal para a prática do exercício físico (antes, durante ou depois) do conteúdo a ser aprendido, como também ao tipo de exercício físico realizado (aeróbio ou anaeróbio), a intensidade aplicada (leve, moderada ou vigorosa) e ao gênero dos participantes 
(masculino ou feminino) para que se possa descrever com maior rigor o papel do exercício físico em relação à produção de BDNF e sua contribuição no processo de aprendizagem.

\section{REFERÊNCIAS}

AICARDI, Giorgio et al. Induction of long-term potentiation and depression is reflected by corresponding changes in secretion of endogenous brain-derived neurotrophic factor. Proceedings of the National Academy of Sciences of the United States of America, v. 101, n. 44, p. 15788-15792, 2004. Disponível em:

https://www.ncbi.nlm.nih.gov/pubmed/15505222. Acesso em: 10 maio 2017.

AMARO, Kassandra Nunes. Intervenção motora para escolares com dificuldade de aprendizagem. 2010. 114 f. Dissertação (Mestrado em Ciências do Movimento Humano) Centro de Ciências da Saúde e do Esporte, Universidade do Estado de Santa Catarina, Florianópolis, 2010.

ARDOY, Daniel Navarro et al. A Physical Education trial improves adolescents' cognitive performance and academic achievement: the EDUFIT study. Scandinavian Journal of Medicine \& Cience in Sports, v. 24, n. 1, 2014. Disponível em:

https://www.ncbi.nlm.nih.gov/pubmed/23826633. Acesso em: 11 maio 2017.

BARDE, Yves-Alain.; EDGAR, David.; THOENEN, Hans. Purification of a new neurotrophic factor from mammalian brain. The EMBO journal, v. 1, n. 5, p. 549-553, 1982. Disponível em: https://www.ncbi.nlm.nih.gov/pmc/articles/PMC553086/. Acesso em: 10 maio 2017.

BAKSHI, Lisa. Will CrossFit Make American Kids Smarter?. CrossFit Journal, p. 1-4, 2008. Disponível em:

http://citeseerx.ist.psu.edu/viewdoc/download?doi=10.1.1.577.3042\&rep=rep1\&type=pd f. Acesso em 12 maio 2017.

BECK, Mikkel M. et al. Motor-enriched learning activities can improve mathematical performance in preadolescent children. Frontiers In Human Neuroscience, v. 10, n. 645, p. 1-14, 2016. Disponível em:

https://www.ncbi.nlm.nih.gov/pmc/articles/PMC5179540/pdf/fnhum-10-00645.pdf. Acesso em: 12 maio 2017.

BEST, Phillip J.; WHITE, Aaron M.; MINAI, Ali. Spatial processing in the brain: the activity of hippocampal place cells. Annual Review of Neuroscience, v. 24, n. 1, p. 459-486, 2001. Disponível em: https://www.ncbi.nlm.nih.gov/pubmed/11283318. Acesso em: 13 maio 2017.

BROWN, Angus M.; TEKKÖK, Selva Baltan.; RANSOM, Bruce R. Energy transfer from astrocytes to axons: the role of CNS glycogen. Neurochemistry International, v. 45, n. 4, p. 529-536, 2004. Disponível em: https://www.ncbi.nlm.nih.gov/pubmed/15186919. Acesso em: 13 maio 2017.

BUGG, Julie M.; HEAD, Denise. Exercise moderates age-related atrophy of the medial temporal lobe. Neurobiology of Aging, v. 32, n. 3, p. 506-514, 2011. Disponível em: https://www.ncbi.nlm.nih.gov/pmc/articles/PMC2891908/pdf/nihms105902.pdf. Acesso em: 16 maio 2017. 
BURGESS, Neil; MAGUIRE, Eleanor A.; O'KEEFE, John. The human hippocampus and spatial and episodic memory. Neuron, v. 35, n. 4, p. 625-641, 2002. Disponível em: https://www.ncbi.nlm.nih.gov/pubmed/12194864. Acesso em: 12 maio 2017.

CASTELLI, Darla M. et al. Physical fitness and academic achievement in third-and fifthgrade students. Journal of Sport and Exercise Psychology, v. 29, n. 2, p. 239-252, 2007. Disponível em: https://www.ncbi.nlm.nih.gov/pubmed/17568069. Acesso em: 13 maio 2017.

CHADDOCK, Laura et al. A neuroimaging investigation of the association between aerobic fitness, hippocampal volume, and memory performance in preadolescent children. Brain research, v. 1358, p. 172-183, 2010. Disponível em:

https://www.ncbi.nlm.nih.gov/pmc/articles/PMC3953557/. Acesso em: 10 maio 2017.

CHADDOCK, Laura. The effects of physical activity on the brain and cognition during childhood. 2013. Tese (Doutorado em Philosophy in Psychology) - University of Illinois, Champaign, 2013.

CHAN, Agnes Suiyin.; CHEUNG, Mei-Chun; SZE, Sophia. Effect of mind/body training on children with behavioral and learning problems: a randomized controlled study. Nova Science Publisher Inc, p. 1-30, 2008. Disponível em: http://neurolab347.pic6.eznetonline.com/upload/21 5qb0.pdf. Acesso em: 17 maio 2017.

CHOMITZ, Virginia R. et al. Is there a relationship between physical fitness and academic achievement? Positive results from public school children in the northeastern United States. Journal of School Health, v. 79, n. 1, p. 30-37, 2009. Disponível em: https://www.ncbi.nlm.nih.gov/pubmed/19149783. Acesso em: 9 maio 2017.

DA SILVA, Cristiane Alves et al. A importância da avaliação motora em escolares. Revista Iberoamericana de Psicomotricidad y Técnicas Corporales, v. 7. n. 26, p. 137-146, 2007. Disponível em: http://www.motricidade.com.br/pdfs/edm/2007.4.pdf. Acesso em: 5 maio 2017.

DELLA-MAGGIORE, Valeria et al. Corticolimbic interactions associated with performance on a short-term memory task are modified by age. Journal of Neuroscience, v. 20, n. 22, p. 8410-8416, 2000. Disponível em:

http://www.jneurosci.org/content/jneuro/20/22/8410.full.pdf. Acesso em: 02 maio 2017.

DE MORAES FERRARI, Gerson Luis et al. Associação da aptidão física e desempenho acadêmico de escolares. Revista Brasileira de Ciência e Movimento, v. 22, n. 4, p. 37-46, 2014. Disponível em: https://portalrevistas.ucb.br/index.php/RBCM/article/view/4954. Acesso em: 4 maio 2017.

DIAS, Marcela Brandão et al. Efeito de brincadeiras ativas sobre o desempenho escolar em crianças. Educação Física em Revista, v. 7, n. 1, 2013. Disponível em:

https://portalrevistas.ucb.br/index.php/efr/article/view/3938. Acesso em: 4 maio 2017.

EPSTEIN, Meryl Heather. Coordinative aerobic exercise does not enhance attention and concentration in college students. 2011. Tese (Mestrado em Sciences in Health and Human Performance, Exercise Science) - University of Montana Missoula, Montana, 2011.

ERICKSON, Kirk I. et al. Exercise training increases size of hippocampus and improves memory. Proceedings of the National Academy of Sciences of USA, v. 108, n. 7, p. 30173022, 2011. Disponível em: http://www.pnas.org/content/108/7/3017. Acesso em: 10 maio 2017. 
FIGUROV, Alexander et al. Regulation of synaptic responses to high-frequency stimulation and LTP by neurotrophins in the hippocampus. Nature, v. 381, n. 6584, p. 706, 1996. Disponível em: https://www.nature.com/articles/381706a0. Acesso em: 7 maio 2017.

FLINT, Jonathan. The genetic basis of cognition. Brain, v. 122, n. 11, p. 2015-2032, 1999. Disponível em: https://www.ncbi.nlm.nih.gov/pubmed/10545388. Acesso em: 6 maio 2017.

GUERRA, Leonor Bezerra; LOPES, Mariana Zaramela; PEREIRA, Alexandre Hatem. Neuroeduca - Inserção da neurobiologia na educação. In: CONGRESSO BRASILEIRO DE EXTENSÃO UNIVERSITÁRIA, 2., Belo Horizonte, 2004. Anais eletrônicos... Belo Horizonte: UFMG, 2004. Disponível em: https://www.ufmg.br/congrext/Educa/Educa113.pdf. Acesso em: 6 maio 2017. HALVORSON, Aslynn Courtney. The impact of acute bouts of two types of physical activity on cognition in elementary school-aged children. 2014. Tese (Mestrado em Science) - University of Tennessee, Knoxville, 2014.

HILLMAN, Charles H.; ERICKSON, Kirk I.; KRAMER, Arthur F. Be smart, exercise your heart: exercise effects on brain and cognition. Nature reviews neuroscience, v. 9, n. 1, p. 58-65, 2008. Disponível em: https://www.nature.com/articles/nrn2298. Acesso em: 2 maio 2017.

HOF, Patrick R; MORRISON, John $\mathrm{H}$. The aging brain: morphomolecular senescence of cortical circuits. Trends in Neurosciences, v. 27, n. 10, p. 607-613, 2004. Disponível em: https://www.sciencedirect.com/science/article/pii/S0166223604002413?via\%3Dihub. Acesso em: 4 maio 2017.

HOPKINS, Michael E. et al. Differential effects of acute and regular physical exercise on cognition and affect. Neuroscience, v. 215, p. 59-68, 2012. Disponível em: https://www.sciencedirect.com/science/article/pii/S0306452212004186?via\%3Dihub. Acesso em: 8 maio 2017.

IZQUIERDO, Iván et al. Mechanisms for memory types differ. Nature, v. 393, p. 635-636, 1998. Disponível em: https://www.nature.com/articles/31371. Acesso em: 3 maio 2017.

KOMULAINEN, Pirjo et al. BDNF is a novel marker of cognitive function in ageing women: the DR's EXTRA Study. Neurobiology of Learning and Memory, v. 90, n. 4, p. 596-603, 2008. Disponível em:

https://www.sciencedirect.com/science/article/pii/S1074742708001287?via\%3Dihub. Acesso em: 7 maio 2017.

KNAEPEN, Kristel et al. Neuroplasticity - exercise-induced response of peripheral brainderived neurotrophic factor. Sports Medicine, v. 40, n. 9, p. 765-801, 2010. Disponível em: https://www.ncbi.nlm.nih.gov/pubmed/20726622. Acesso em: 2 maio 2017.

LABBAN, Jeffrey D.; ETNIER, Jennifer L. Effects of acute exercise on long-term memory. Research Quarterly For Exercise And Sport, v. 82, n. 4, p. 712-721, 2011. Disponível em: https://www.ncbi.nlm.nih.gov/pubmed/22276413. Acesso em: 16 maio 2017.

LISTA, Ilaria.; SORRENTINO, Giuseppe. Biological mechanisms of physical activity in preventing cognitive decline. Cellular and Molecular Neurobiology, v. 30, n. 4, p. 493503, 2010. Disponível em: https://link.springer.com/article/10.1007\%2Fs10571-0099488-x. Acesso em: 7 maio 2017. 
LÖRINCZ, András; BUZSÁKI, György. Two-phase computational model training long-term memories in the entorhinal-hippocampal region. In: Annals of The New York Academy of Sciences, 2006, New York. Anal eletrônico... New York: NYAS. Disponível em: https://www.ncbi.nlm.nih.gov/pubmed/10911869. Acesso em: 9 maio 2017.

MORALES-MIRA, Marco; MAURICIO, Valenzuela-Harrington. Ejercicio físico: su rol en la neurogénesis inducida por bdnf y vegf exercise: your role in neurogenesis induced by bdnf and vegf. Revista Motricidad Humana, v. 15, n. 2, p. 134-142, 2014. Disponível em: https://www.revistamotricidad.com/wp-content/uploads/2015/03/134-142.pdf. Acesso em: 9 maio 2017.

MOTA, João et al. Atividade Física e Rendimento Académico-Uma revisão sistemática de sete revisões sistemáticas. Revista da Sociedade Científica de Pedagogia do Desporto, v. 1, n. 1, p. 24-29, 2015. Disponível em: http://rbafs.org.br/RBAFS/article/view/12120. Acesso em: 11 maio 2017.

PAN, Weihong. et al. Transport of brain-derived neurotrophic factor across the bloodbrain barrier. Neuropharmacology, v. 37, n. 12, p. 1553-1561, 1998. Disponível em: https://www.ncbi.nlm.nih.gov/pubmed/9886678. Acesso em: 5 maio 2017.

PEDERSEN, Bente K. et al. Role of exercise-induced brain-derived neurotrophic factor production in the regulation of energy homeostasis in mammals. Experimental Physiology, v. 94, n. 12, p. 1153-1160, 2009. Disponível em: https://www.ncbi.nlm.nih.gov/pubmed/19748969. Acesso em: 9 maio 2017.

POO, Mu-ming. Neurotrophins as synaptic modulators. Nature Reviews Neuroscience, v. 2, n. 1, p. 24-32, 2001. Disponível em: https://www.nature.com/articles/35049004. Acesso em: 19 maio 2017.

RASMUSSEN, Peter et al. Evidence for a release of brain-derived neurotrophic factor from the brain during exercise. Experimental Physiology, v. 94, n. 10, p. 1062-1069, 2009. Disponível em: https://www.ncbi.nlm.nih.gov/pubmed/19666694. Acesso em: 8 maio 2017.

REED, Julian A. et al. Examining the impact of 45 minutes of daily physical education on cognitive ability, fitness performance, and body composition of African American youth. Journal of Physical Activity and Health, v. 10, n. 2, p. 185-197, 2013. Disponível em: https://journals.humankinetics.com/doi/10.1123/jpah.10.2.185. Acesso em: 9 maio 2017.

RUMAJOGEE, Prakasham et al. Adaption of the serotoninergic neuronal phenotype in the absence of 5-HT autoreceptors or the 5-HT transporter: involvement of BDNF and cAMP. European Journal of Neuroscience, v. 19, n. 4, p. 937-944, 2004. Disponível em: https://www.ncbi.nlm.nih.gov/pubmed/15009141. Acesso em: 9 maio 2017.

SEIFERT, Thomas et al. Endurance training enhances BDNF release from the human brain. American Journal of Physiology, Regulatory, Integrative and Comparative Physiology, v. 298, n. 2, p. 372-377, 2009. Disponível em:

https://www.ncbi.nlm.nih.gov/pubmed/19923361. Acesso em: 17 maio 2017.

SILVÉRIO, Gustavo Camargo; ROSAT, Renata Menezes. Memória de Longo Prazo: Mecanismos Neurofisiológicos de Formação. Revista Médica de Minas Gerais, v.16, n. 4, p. 219-23, 2006. Disponível em: http://rmmg.org/artigo/detalhes/577. Acesso em: 17 maio 2017.

SO, Wi-Young. Association between physical activity and academic performance in Korean adolescent students. BMC Public Health, v. 12, n. 1, p. 258, 2012. Disponível em: 
https://bmcpublichealth.biomedcentral.com/articles/10.1186/1471-2458-12-258. Acesso em: 15 maio 2017.

SCHMIDT-KASSOW, Maren et al. Exercising during learning improves vocabulary acquisition: behavioral and ERP evidence. Neuroscience Letters, v. 482, n. 1, p. 40-44, 2010. Disponível em: https://www.ncbi.nlm.nih.gov/pubmed/20620190. Acesso em: 15 maio 2017.

SKRADE, Miriam Ann Blom. Integrated classroom physical activity: Examining perceived need satisfaction and academic performance in children. 2013. Tese (Mestrado em Sciences) - Iowa State University, Iowa, 2013.

SKRIVER, Kasper et al. Acute exercise improves motor memory: exploring potential biomarkers. Neurobiology of Learning and Memory, v. 116, p. 46-58, 2014. Disponível em: https://www.ncbi.nlm.nih.gov/pubmed/25128877. Acesso em: 7 maio 2017. SPITZER, Ursula S; HOLLMANN, Wildor. Experimental observations of the effects of physical exercise on attention, academic and prosocial performance in school settings. Trends in Neuroscience and Education, v. 2, n. 1, p. 1-6, 2013. Disponível em: https://www.sciencedirect.com/science/article/pii/S2211949313000045. Acesso em: 08 maio 2017.

SZUHANY, Kristin L.; BUGATTI, Matteo.; OTTO, Michael W. A meta-analytic review of the effects of exercise on brain-derived neurotrophic factor. Journal of Psychiatric Research, v. 60, p. 56-64, 2015. Disponível em: https://www.ncbi.nlm.nih.gov/pubmed/25455510. Acesso em: 03 maio 2017.

TANAKA, Jun-Ichi et al. Protein synthesis and neurotrophin-dependent structural plasticity of single dendritic spines. Science, v. 319, n. 5870, p. 1683-1687, 2008. Disponível em: https://www.ncbi.nlm.nih.gov/pubmed/18309046. Acesso em: 17 maio 2017.

VAN DEN BERG, Vera et al. Physical activity in the school setting: cognitive performance is not affected by three different types of acute exercise. Frontiers in Psychology, v. 7, p. 723, 2016. Disponível em: https://www.ncbi.nlm.nih.gov/pubmed/27242629. Acesso em: 9 maio 2017.

ZHAO, Wei-Qin et al. Insulin and the insulin receptor in experimental models of learning and memory. European Journal of Pharmacology, v. 490, n. 1-3, p. 71-81, 2004. Disponível em: https://www.ncbi.nlm.nih.gov/pubmed/15094074. Acesso em: 10 maio 2017.

WILLIAMS, Graham V.; RAO, Srinivas G.; GOLDMAN-RAKIC, Patricia S. The physiological role of 5-HT2A receptors in working memory. Journal of Neuroscience, v. 22, n. 7, p. 2843-2854, 2002. Disponível em: https://www.ncbi.nlm.nih.gov/pubmed/11923449. Acesso em: 10 maio 2017.

WINTER, Bernward et al. High impact running improves learning. Neurobiology of Learning and Memory, v. 87, n. 4, p. 597-609, 2007. Disponível em: https://www.ncbi.nlm.nih.gov/pubmed/17185007. Acesso em: 6 maio 2017.

WOOLF, Nancy J. et al. Elevation of nerve growth factor and antisense knockdown of TrkA receptor during contextual memory consolidation. Journal of Neuroscience, v. 21, n. 3, p. 1047-1055, 2001. Disponível em:

https://www.ncbi.nlm.nih.gov/pubmed/11157090. Acesso em: 12 maio 2017. 